Corrigendum

\title{
Corrigendum to "Safety and Efficacy of Small Bowel Examination by Capsule Endoscopy for Patients before Liver Transplantation"
}

\author{
Seiji Kawano, ${ }^{1}$ Akinobu Takaki, ${ }^{1}$ Masaya Iwamuro, ${ }^{1}$ Tetsuya Yasunaka, ${ }^{1}$ \\ Yoshiyasu Kono, ${ }^{1}$ Kou Miura, ${ }^{1}$ Toshihiro Inokuchi, ${ }^{1}$ Yoshiro Kawahara, ${ }^{2}$ \\ Yuzo Umeda, ${ }^{3}$ Takahito Yagi, ${ }^{3}$ and Hiroyuki Okada ${ }^{1}$ \\ ${ }^{1}$ Department of Gastroenterology and Hepatology, Okayama University Graduate School of Medicine, \\ Dentistry, and Pharmaceutical Sciences, Okayama, Japan \\ ${ }^{2}$ Department of Endoscopy, Okayama University Hospital, Okayama, Japan \\ ${ }^{3}$ Department of Hepato-Biliary-Pancreatic Surgery, Okayama University Hospital, Okayama, Japan \\ Correspondence should be addressed to Seiji Kawano; skawano@mpd.biglobe.ne.jp
}

Received 6 April 2017; Accepted 9 May 2017; Published 3 July 2017

Copyright (C) 2017 Seiji Kawano et al. This is an open access article distributed under the Creative Commons Attribution License, which permits unrestricted use, distribution, and reproduction in any medium, provided the original work is properly cited.

In the article titled "Safety and Efficacy of Small Bowel Examination by Capsule Endoscopy for Patients before Liver Transplantation" [1], the first and last names of all the authors were reversed. The revised authors' list is shown above.

\section{References}

[1] K. Seiji, T. Akinobu, and I. Masaya et al., "Safety and efficacy of small bowel examination by capsule endoscopy for patients before liver transplantation," BioMed Research International, vol. 2017, Article ID 8193821, 6 pages, 2017. 


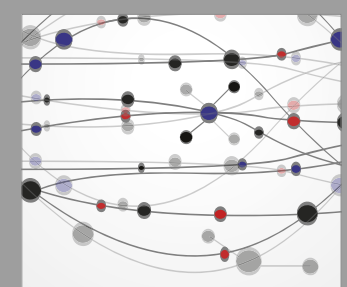

The Scientific World Journal
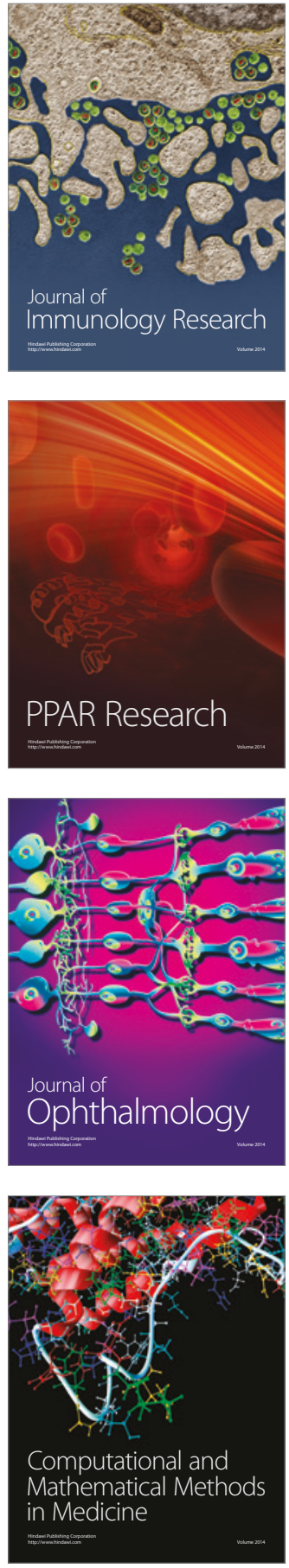

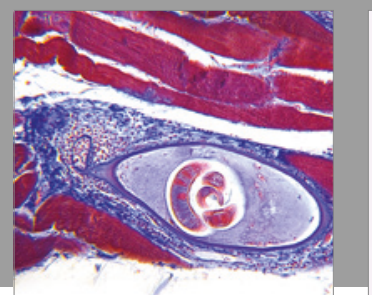

Gastroenterology Research and Practice
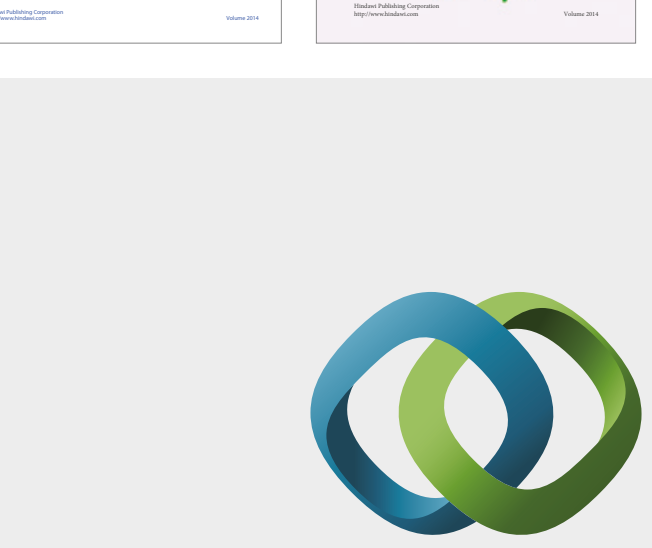

\section{Hindawi}

Submit your manuscripts at

https://www.hindawi.com
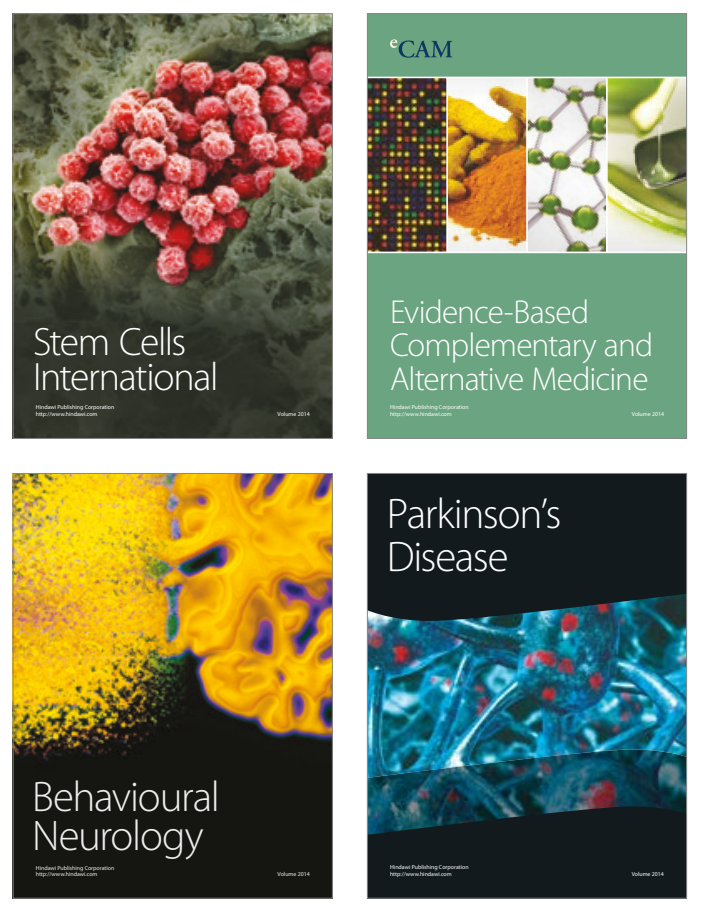
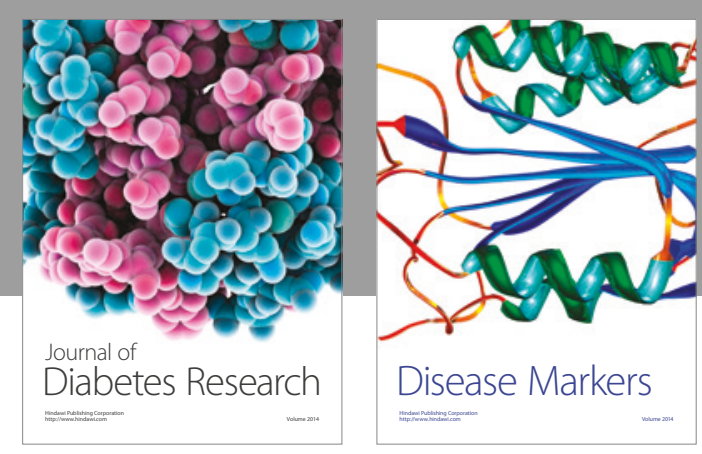

Disease Markers
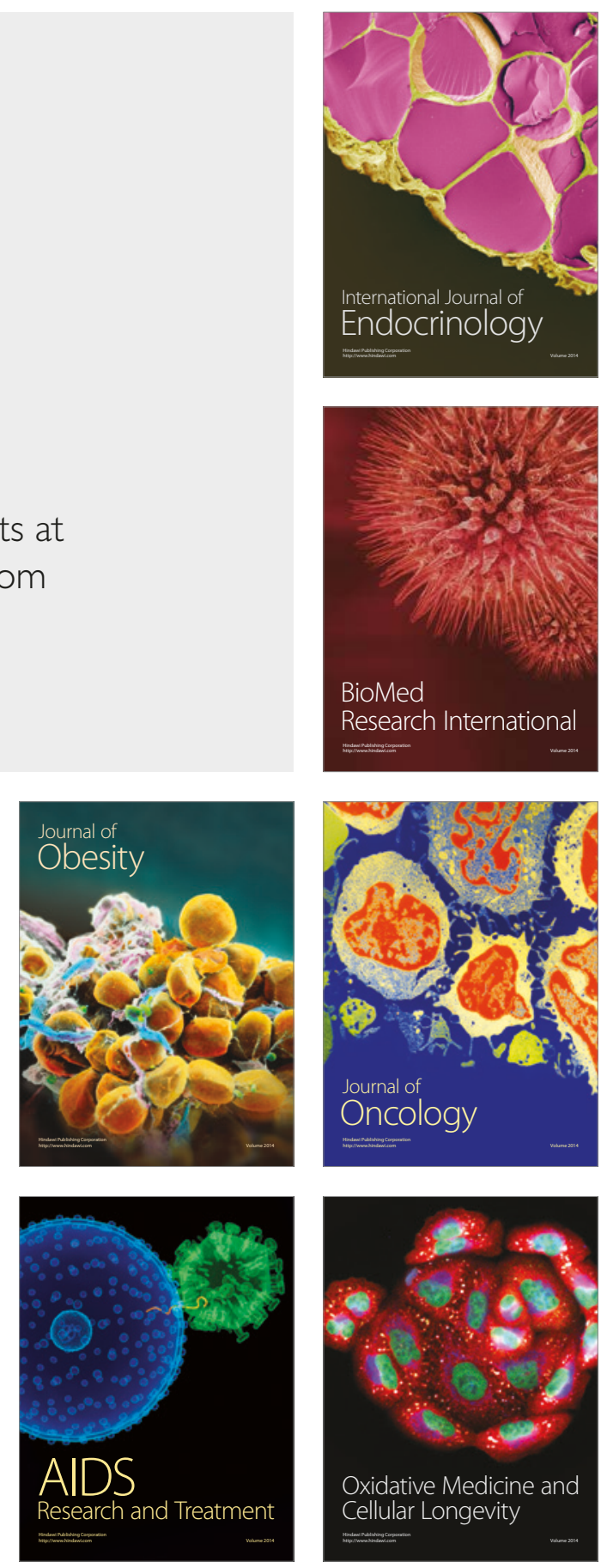